\title{
¿Ha crecido la clase media en el Ecuador? Un análisis mediante índices de polarización del ingreso para el periodo 2007-2014
}

Recibido: septiembre, 14 de 2017 - Aprobado: abril, 2 de 2018

Doi: http://dx.doi.org/10.12804/revistas.urosario.edu.co/economia/a.6802

\author{
Esteban Cabrera Cevallos*
}

Edwin Buenaño ${ }^{\dagger}$

\section{Resumen}

En este artículo se cuantifica la evolución de la clase media en el Ecuador entre el 2007 y el 2014, mediante el cálculo de índices de polarización y bipolarización del ingreso. Los resultados muestran que durante el periodo de análisis la polarización disminuyó de manera constante, debido a un desplazamiento homogéneo de los hogares de clases más bajas hacia clases más altas, lo que se traduce en un incremento de la clase media, cuantificada como proporción de la población total.

Palabras clave: polarización, bipolarización, clase media, desigualdad.

Clasificación JEL: A33, D31, D63, D74

Los autores agradecen los comentarios de Fausto Jácome y Paúl Carrillo.

Las opiniones, errores y omisiones son responsabilidad exclusiva de los autores y no necesariamente reflejan la posición oficial del INEC o de la PUCE, ni de sus autoridades.

* Economista de la Pontificia Universidad Católica del Ecuador - PUCE-. Analista de Innovación en Métricas y Metodologías en el Instituto Nacional de Estadística y Censos _-INEC—,Ecuador. Correos electrónicos: carlos_cabrera@inec.gob.ec, cecc_1593@hotmail. com

† Economista de la Pontificia Universidad Católica del Ecuador - PUCE-. Máster en Estadística Aplicada de la Escuela Politécnica Nacional - EPN-. Máster en Economía Aplicada de la Universidad Autónoma de Barcelona - UAB-. Doctor en Economía Aplicada por la UAB. Profesor de Econometría en la Facultad de Economía de la PUCE. Correo electrónico: evbuenano@puce.edu.ec

Cómo citar este artículo: Cabrera Cevallos, E. \& Buenaño, E. (2018). ¿Ha crecido la clase media en el Ecuador? Un análisis mediante índices de polarización del ingreso para el periodo 2007-2014. Revista de Economía del Rosario, 21(1), 121-152.

Doi: http://dx.doi.org/10.12804/revistas.urosario.edu.co/economia/a.6802 


\title{
Has the Middle Class Grown in Ecuador? An Analysis Using Income Polarization Indexes for the Period 2007 to 2014
}

\begin{abstract}
This paper quantifies the evolution of the middle class in Ecuador between 2007 and 2014 by computing polarization and bipolarization indexes. The results show that polarization have steadily decline between 2007 and 2014 due to an homogeneous move from lower income class households to higher income classes, which translates into an increase of the middle class measured as proportion of the total population.
\end{abstract}

Keywords: polarization, bipolarization, middle class, inequality.

JEL Classification: A33, D31, D63, D74

\section{Tem crescido a classe média no Equador? Uma análise mediante índices de polarização para o período 2007-2014}

\section{Resumo}

Neste artigo quantifica-se a evolução da classe média no Equador entre o ano 2007 e o ano 2014, mediante o cálculo de índices de polarização e bipolarização. Os resultados mostram que durante o período de análise a polarização diminuiu de maneira constante, devido a um deslocamento homogéneo dos lares de classes mais baixas para as classes mais altas, o que se traduz em um incremento da classe média, quantificada como proporção da população total.

Palavras-chave: polarização, bipolarização, classe média, desigualdade.

Classificação JEL: A33, D31, D63, D74 


\section{Introducción}

En el Ecuador, según datos del Instituto Nacional de Estadística y Censos —INEC—, entre el 2007 y el 2014, la pobreza por ingresos se redujo $13 \%$, pasando de $36,7 \%$ a $22,5 \%$. En el área rural la reducción fue de $61,3 \%$ a $35,3 \%$, mientras que en el área urbana pasó de $24,3 \%$ a $16,4 \%$. En cuanto a la desigualdad medida por el índice de Gini, esta se redujo en 8 puntos, pasando de 0,55 en el 2007 a 0,47 en el 2014 a nivel nacional.

Distintos autores, como el Banco Mundial (2014), Cruces, Fields, Jaume y Viollaz (2015) y Cuevas, Atuesta y Zanbonino (2016), coinciden con ese diagnóstico y determinan que algunos factores que contribuyeron a ese proceso de redistribución del ingreso durante el periodo de análisis fueron el ingreso laboral y las transferencias públicas. Según Cuevas et al. (2016), el ingreso laboral se incrementó en todos los grupos socioeconómicos, pero más entre los percentiles más bajo de la distribución.

No obstante, los cambios en la desigualdad no implican, necesariamente, cambios en los polos del ingreso. Por ello, para un país que ha conseguido importantes mejoras en términos de pobreza y desigualdad se torna relevante profundizar en el análisis de la distribución del ingreso, observando no solo sus índices de desigualdad sino también el debilitamiento o el fortalecimiento del grupo de ingresos medios frente al de sus extremos alto y bajo.

El análisis de la clase media resulta relevante ya que constituye un factor importante en el crecimiento y el desarrollo en las economías industrializadas (Foster y Wolfson 1992). Varios investigadores, como Acemoglu y Zilibotti (1997), Doepke y Zilibotti (2005), Banerjee y Duflo (2008), Solimano (2008), Kharas (2010), Nayab (2011), entre otros, han concluido que la clase media es un estrato especial en la sociedad, pues aumenta su estabilidad y reduce la percepción de desigualdad entre ricos y pobres. Además, la clase media está asociada a características que dan cuenta de una mejora en el capital humano, como personas con mayor nivel de educación, mayor capacidad de ahorro, emprendedoras, entre otros aspectos. Por lo tanto, el progreso de este estrato social puede llegar a ser un factor esencial para el desarrollo económico.

No obstante, la medición de la clase media reviste algunas complicaciones: a diferencia del estudio de la pobreza monetaria, el estudio de los estratos medios requiere definir tanto umbrales inferiores como superiores, pues deben distinguirse tres grupos sociales. Si bien existe un amplio abanico de propuestas metodológicas que la literatura ha desarrollado para la medición de este grupo social, también existe un amplio debate alrededor de estas técnicas.

Como alternativa a las metodologías tradicionales surgen las propuestas basadas en los índices de polarización, los cuales forman parte de lo que se 
conoce como medidas endógenas de clase media. Tienen la característica de que determinan los umbrales que dividen a las distintas clases sociales con base en la distribución del ingreso. Cabe señalar que la polarización es un concepto que está muy relacionado con la desigualdad pero que se desenvuelve en una dimensión diferente. Por un lado, la polarización capta la formación de grupos o polos antagónicos en una distribución, mientras que la desigualdad se centra en la dispersión global de la distribución. Por ello, los análisis de desigualdad y polarización, en ocasiones, se suelen utilizar de forma complementaria.

La profundización en el estudio de este tema data de los años noventa por la percepción del decremento de la clase media estadounidense en la década de los ochenta y la crítica a su medición, efectuada por investigadores con intereses afines con el crecimiento económico, la convergencia y la desigualdad (véase Blackburn \& Bloom, 1985; Thurow, 1984; Foster \& Wolfson, 1992). Posteriormente, los estudios de polarización abarcaron más que cuestiones puramente monetarias, pues el concepto se extiende a otras características clave en el desarrollo de un país, como la estabilidad y la tensión social. Así, Borraz, González y Rossi (2013) mencionan que el grado de polarización de una sociedad es un factor importante para el desarrollo económico.

La definición formal de la polarización es tratada por Esteban y Ray (1994), quienes integran interpretaciones de distancias sociales en términos de atributos ideológicos y económicos. Bajo este enfoque, el concepto de polarización está estrechamente ligado a la generación de tensión social y conflicto, y en general a la existencia de malestar social. En la literatura, es común que el marco de identificación-alienación, como se conoce al desarrollo de Esteban y Ray (1994), sea el predominante al hablar de polarización, pues se podría considerar que el enfoque de bipolarización corresponde a un caso particular del indicador propuesto por Esteban, Gradín y Ray (2007).

La evidencia empírica en la región sobre análisis de polarización es limitada. Algunos autores que han estudiado el tema son Gradín y Rossi (2000), quienes observan la desigualdad y la bipolarización del salario en Uruguay y notan que la distribución de esa variable es progresivamente más desigual, pero sobretodo más bipolarizada, debido, principalmente, a las diferencias salariales por rama. Por su parte, Huesca (2003) realiza un análisis de la polarización del ingreso de los hogares en México entre 1984 y el 2000, en el que concluye que la polarización en dicho país se incrementó y se produjo una pérdida relativa del peso de la clase media. En otro estudio para la región, Gasparini, Horenstein, Molina y Olivieri (2008) realizan un análisis para los países de América Latina y el Caribe (1989-2004), encontrando que existe una correlación positiva entre los niveles de polarización y los niveles de conflicto 
social. En la misma línea, Larrú (2013) analiza la desigualdad, la polarización y la ayuda al desarrollo para 18 países de América Latina durante los años noventa, encontrando que la polarización está asociada positivamente con el gasto público y las remesas pero negativamente con las transferencias condicionadas.

En relación con la clase media, Borraz et al. (2013) argumentan que los índices de polarización están fuertemente relacionados al tamaño de la clase media, ya que su incremento o disminución está atado a cambios en los grupos de población de ricos o pobres. Según Ferreira et al. (2013), la población de clase media en América Latina y el Caribe aumentó en un 50\%, de 103 millones de personas en el 2003 a 152 millones en el 2009. Sin embargo, el panorama es menos homogéneo en cuanto a polarización; al analizar el estudio de Gasparini, Horenstein, y Olivieri (2006), se observa que, en promedio, la polarización del ingreso aumentó ligeramente (2,5 puntos porcentuales) en la región desde principios de 1990 hasta el 2004.

En el caso ecuatoriano no existen estudios que analicen la clase media a partir de medidas endógenas como las utilizadas en la presente investigación. Por ello, el interés central del estudio consiste en estimar, mediante varios índices, la evolución de la polarización y la de la clase media en el Ecuador para el periodo 2007-2014.

En lo que resta del documento, la estructura que se presenta es la siguiente: en la segunda parte se hace una breve revisión a la literatura sobre medición de la clase media; en la tercera parte se muestra la metodología empleada para la estimación de los diferentes índices utilizados en el análisis; la cuarta parte contiene la descripción de los datos junto con el análisis y la discusión de los resultados; finalmente, se presentan las conclusiones de la investigación.

\section{Medición de la clase media}

La medición de la clase media es un tema que reviste cierta polémica y dificultad debido a la multiplicidad de esquemas existentes y a sus limitaciones (Pendfold \& Rodríguez, 2014). A diferencia del estudio de la pobreza monetaria, el análisis de los estratos medios requiere definir un umbral superior que divida a la clase media de los ricos (Edo \& Sosa-Escudero, 2013).

Cruces, López-Calva y Battiston (2010) realizan una amplia revisión de las definiciones usadas en la literatura para distinguir a la clase media. Se distinguen cinco grupos de definiciones: 1) basadas en medidas de tendencia central; 2) basadas en cuantiles; 3) basadas en umbrales absolutos (líneas de pobreza); 4) basadas en estrategias mixtas; y 5) endógenas. Siguiendo a Olivieri (2007), 
los primeros cuatro grupos de definiciones son medidas "exógenas", pues consisten en una definición previa $(a d h o c)$ de lo que se entenderá por clase social, mientras que en las definiciones "endógenas", los puntos de corte de las distintas clases sociales no están previamente determinadas.

\subsection{Medidas exógenas}

El uso de medidas exógenas para cuantificar a la clase media es predominante en la literatura. Algunos autores, como Ferreira et al. (2013), López-Calva y Ortiz-Juarez $(2012,2013)$, entre otros, clasifican a las medidas exógenas en tres clases: 1) medidas relativas, las cuales identifican a la clase media como aquellos hogares con un nivel de consumo o ingreso dentro de un rango específico alrededor de la mediana de la distribución; 2) medidas absolutas, las cuales se caracterizan por ser umbrales absolutos respecto al consumo o al ingreso, generalmente ligados a los umbrales de medición de pobreza monetaria a nivel internacional; y 3) medidas mixtas, cuyo umbral inferior es absoluto y su umbral superior es relativo a la distribución.

La tabla 1 presenta un resumen de las principales definiciones exógenas desarrolladas en la literatura de clase media. Las definiciones relativas se presentan en términos de percentiles de la distribución, donde $P_{r}$ es el percentil $n$ de la distribución y $y_{i}$ el ingreso percapita del hogar; por otro lado, en las definiciones absolutas todos los valores se expresan en US\$ convertidos al tipo de cambio paridad de poder adquisitivo - PPA-; finalmente, las definiciones mixtas son una combinación de ambas.

\subsection{Medidas endógenas y polarización}

Las medidas endógenas se caracterizan por determinar los umbrales que dividen a las distintas clases sociales con base en las características de la distribución del ingreso, para lo cual se han desarrollado estudios no paramétricos (véase Zhu, 2005; Massari, Pittau \& Zelli, 2009; D'Ambrosio, Muliere \& Secchi, 2002; Olivieri, 2007). Entre estas medidas se encuentran los indicadores de polarización, los cuales están estrechamente ligados a variaciones en el tamaño de los estratos medios y la cohesión económica, debido a que captan la formación de polos o grupos en la distribución del ingreso.

Según Esteban y Ray (1994), la polarización de una distribución de atributos (ej.: ingreso per cápita) individuales debe cumplir con las siguientes características: 1) alto grado de homogeneidad dentro del grupo (identificación); 2) alto grado de heterogeneidad entre grupos (alienación); y 3) número 
Tabla 1. Definiciones comunes de clase media con base en el ingreso o el consumo: enfoque relativo, absoluto y mixto

\begin{tabular}{|c|c|c|c|}
\hline Enfoque & Tipo & Autores & Definición \\
\hline \multirow{8}{*}{ 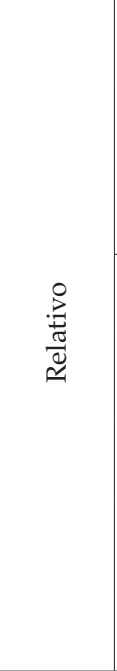 } & \multirow{3}{*}{$\begin{array}{l}\text { Basadas en } \\
\text { medidas de } \\
\text { tendencia } \\
\text { central }\end{array}$} & $\begin{array}{l}\text { Blackburn \& } \\
\text { Bloom (1985) }\end{array}$ & $i \in C M \Leftrightarrow 0,6 y\left(P_{50}\right) \leq y_{i} \leq 2,25 y\left(P_{50}\right)$ \\
\hline & & Thurrow (1987) & $i \in C M \Leftrightarrow 0,75 y\left(P_{50}\right) \leq y_{i} \leq 1,25 y\left(P_{50}\right)$ \\
\hline & & $\begin{array}{l}\text { Davis \& Hudson } \\
\quad(1992)\end{array}$ & $i \in C M \Leftrightarrow 0,5 y\left(P_{50}\right) \leq y_{i} \leq 1,5 y\left(P_{50}\right)$ \\
\hline & \multirow{5}{*}{$\begin{array}{l}\text { Basadas en } \\
\text { percentiles }\end{array}$} & Levy (1987) & $i \in C M \Leftrightarrow P_{20} \leq y_{i} \leq P_{80}$ \\
\hline & & $\begin{array}{l}\text { Alesina \& Perotti } \\
\text { (1996) }\end{array}$ & $i \in C M \Leftrightarrow P_{40} \leq y_{i} \leq P_{80}$ \\
\hline & & Patridge (1997) & $i \in C M \Leftrightarrow P_{50} \leq y_{i} \leq P_{60}$ \\
\hline & & $\begin{array}{l}\text { Barro (1998) y } \\
\text { Easterly (2001) }\end{array}$ & $i \in C M \Leftrightarrow P_{30} \leq y_{i} \leq P_{80}$ \\
\hline & & Solimano (2008) & $i \in C M \Leftrightarrow P_{30} \leq y_{i} \leq P_{90}$ \\
\hline \multirow{4}{*}{$\begin{array}{l}0 \\
\frac{0}{3} \\
0 \\
0 \\
\frac{0}{<}\end{array}$} & \multirow{4}{*}{$\begin{array}{l}\text { Basadas en } \\
\text { umbrales } \\
\text { absolutos }\end{array}$} & $\begin{array}{l}\text { Banarjee \& Duflo } \\
\qquad(2008)\end{array}$ & $i \in C M \Leftrightarrow \$ 2 \leq y_{i} \leq \$ 10$ aldia \\
\hline & & Kharas (2010) & $i \in C M \Leftrightarrow \$ 10 \leq y_{i} \leq \$ 100$ aldia \\
\hline & & Ravallion (2010) & $i \in C M \Leftrightarrow \$ 2 \leq y_{i} \leq \$ 13$ aldia \\
\hline & & $\begin{array}{c}\text { López-Calva \& } \\
\text { Ortiz-Juarez (2013) }\end{array}$ & $i \in C M \Leftrightarrow \$ 10 \leq y_{i} \leq \$ 50$ aldia \\
\hline \multirow{2}{*}{ 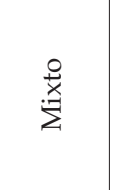 } & \multirow{2}{*}{$\begin{array}{l}\text { Basado en } \\
\text { umbrales abso- } \\
\text { lutos y medidas } \\
\text { de tendencia } \\
\text { central }\end{array}$} & $\begin{array}{l}\text { Sosa-Escudero \& } \\
\text { Petralia (2010) }\end{array}$ & $i \in C M \Leftrightarrow \$ L P \leq y_{i} \leq P_{90}$ \\
\hline & & Birdsall (2010) & $i \in C M \Leftrightarrow \$ 10 \leq y_{i} \leq P_{90}$ \\
\hline
\end{tabular}

Fuente: elaboración propia basada en Cruces et al. (2010), Ferreira et al. (2013), López-Calva y Ortiz-Juarez (2012)

pequeño de grupos de tamaño significativo. De esta forma, la sociedad podrá dividirse en grupos cuyos individuos son "muy parecidos" dentro de un grupo (homogeneidad intragrupo), y se encuentran "distantes" entre grupos (heterogeneidad entregrupos). Según Viollaz (2008), los tres elementos, identificación, distanciamiento y tamaño del grupo, producen antagonismo entre la población y generan conflicto. Por tanto, la polarización hace referencia a situaciones de antagonismo entre grupos internamente homogéneos pero claramente diferenciados en término de ingresos. 
Así como las fuerzas de la identificación y la alienación ayudan a explicar la dimensión de la polarización, también lo hacen al momento de diferenciarla de la desigualdad. Por un lado, la polarización capta la formación de grupos o polos antagónicos en una distribución, mientras que, por otro, la desigualdad se centra en la dispersión global de la distribución. Como lo mencionan, el análisis de la polarización debe ser visto como complementario al de la desigualdad, ya que ambas son dimensiones diferentes pero relacionadas con una misma distribución.

\section{Indicadores de polarización}

Las raíces económicas de la polarización recaen en los trabajos de Foster y Wolfson (1992), Wolfson (1994) y Esteban y Ray (1994). Por un lado, el enfoque desarrollado por Wolfson (1994) es una medida de bipolarización, entendida también como una medida de concentración alrededor de la mediana, en la cual la población se divide en dos grupos: aquellos con ingreso menor a la mediana $m$ y aquellos con ingreso superior. El trabajo de Foster y Wolfson (1992) exclusivamente se encarga de estudiar el debilitamiento de la clase media utilizando un enfoque libre de rangos para medir la clase media y la polarización basado en ordenamientos parciales. Su enfoque radica en construir una definición axiomática de un indicador de bipolarización para medir el debilitamiento de la clase media.

Por otro lado, Esteban y Ray (1994), y posteriores extensiones (Duclos, Esteban \& Ray 2004; Esteban et al., 2007), proponen un esquema en el cual la población se podría dividir en más de dos grupos. Su enfoque busca integrar interpretaciones de distancias sociales en términos de atributos ideológicos y económicos. Bajo esta perspectiva, el concepto de polarización está estrechamente ligado a la generación de tensión social y conflicto, y, en general, a la existencia de malestar social. En la literatura, es común que el marco de identificación-alienación sea el predominante al hablar de polarización. Este enfoque, a pesar de no ser diseñado explícitamente para el estudio de los estratos medios, también permite utilizarlo para dividir la distribución en grupos y definir, de este modo, a la clase media de un área geográfica determinada. Cruces et al. (2010) recomiendan su uso para dividir a la poblacion en tres clases, baja, media y alta, argumentando que la medida proporcionada por el indicador de polarización goza de mayor estabilidad frente a la amplia volatibilidad de las medidas con umbrales absolutos. De igual manera, el particionamiento de la sociedad bajo este método presenta homogenidad con los niveles de otras variables sociodemograficas importantes, como los niveles de escolaridad y la situacion en el mercado laboral. 
La tabla 2 presenta un resumen de las principales definiciones de indicadores de polarización desarrolladas en la literatura.

Tabla 2. Indicadores de polarización pura del ingreso

\begin{tabular}{llll}
\multicolumn{1}{c}{ Marco } & \multicolumn{1}{c}{ Autores } & Siglas & \multicolumn{1}{c}{ Descripción } \\
\hline & Esteban \& Ray (1994) & ER & Grupos finitos \\
$\begin{array}{l}\text { Alienación } \\
\text { identificación }\end{array}$ & $\begin{array}{l}\text { Esteban, Gradín y Ray } \\
(1994,2007)\end{array}$ & EGR & $\begin{array}{l}\text { Grupos finitos definidos endógena- } \\
\text { mente }\end{array}$ \\
& Duclos, Esteban y Ray (2004) & DER & $\begin{array}{l}\text { Identifación mediante técnicas no para- } \\
\text { métricas (no grupos finitos de ingreso) }\end{array}$ \\
\hline Bipolarización & Foster \& Wolfson (1992) & W & $\begin{array}{l}\text { Polarización en función del aumento } \\
\text { de la bipolarización }\end{array}$ \\
& Wolfson (1994) & &
\end{tabular}

Fuente: elaboración propia basada en Duclos et al. (2004), Esteban et al. (2007), Esteban y Ray (1994), Foster y Wolfson, 1992

Según Esteban y Ray (1994), una sociedad polarizada es aquella que, con base en un vector de atributos distribuidos en toda la sociedad, se encuentra agrupada en clusters, polos o grupos de tamaños significativos. En este sentido, cada grupo es similar a otro cuando los atributos de sus miembros son semejantes y son diferentes cuando los atributos de sus miembros son disimiles. Por lo general, el vector de atributos utilizado en el estudio de polarización económica es el ingreso per cápita de los individuos, a lo cual se lo denomina la polarización pura del ingreso. No obstante, existen extensiones hacia otras dimensiones, como variables socioeconómicas (véase Zhang \& Kanbur, 2001; Gradín, 2000) o ideologías religiosas (Reynal-Querol, 2002).

\section{Metodología}

\subsection{Polarización}

Se parte de la propuesta de Esteban y Ray (1994), quienes utilizan el logaritmo neperiano del ingreso y para establecer una medida genérica de polarización mediante el enfoque alineación-identificación, tal que:

$$
P=\sum_{i=1}^{n} \sum_{j=1}^{n} \pi_{i} \pi_{j} T\left(I\left(\pi_{i}\right), A\left(\left|\mu_{i}-\mu_{j}\right|\right)\right)
$$

donde $\left(\Pi_{i}\right)$ es una función creciente de la proporción de individuos dentro de un determinado grupo, $A$ representa la alienación calculada en función de la 
distancia absoluta de los ingresos entre grupos, y T(I,A) representa una función de antagonismo efectivo. Por lo tanto, la polarización total $P$ se calcula como el promedio ponderado de cada uno de los antagonismos efectivos. Los autores determinan que el índice de polarización que respeta un conjunto deseable de axiomas (véase Esteban y Ray, 1994) debe ser proporcional a:

$$
P_{E R}\langle\alpha, p\rangle=K \sum_{i=1}^{n} \sum_{j=1}^{n} \pi_{i}^{1+\alpha} \pi_{j}\left|\mu_{i}-\mu_{j}\right|
$$

donde $\mathrm{k}>0$ y $\alpha \in[0 ; 1,6]$ es un parámetro el cual indica el grado de sensibilidad a la identificación.

Sin embargo, el índice $P_{E R}$ (o simplemente ER) asume que la población se encuentra preagrupada, por ello los autores proponen una metodología para determinar endógenamente el tamaño de los grupos, minimizando la heterogeneidad intergrupal. Para ello fijan exógenamente el número de grupos para luego escoger puntos de la distribución que minimicen la dispersión del ingreso dentro de los grupos. Véase tal que:

$$
P_{E G R}(f ; \alpha, \beta)=P_{E R}(\alpha, p)-\beta \varepsilon(f, p)
$$

El primer término de la ecuación (3) corresponde al índice $P_{E R^{\prime}}$ mientas que el segundo término representa una medición del error o falta de identificación, ponderado por un parámetro libre $\beta$. El error se mide como la diferencia entre el coeficiente de Gini al tomar toda la distribución G(f) y el Gini que resulta de separar la población en $n$ grupos; es decir:

$$
\varepsilon\left(f, p^{*}\right)=G(f)-G\left(p^{*}\right)
$$

Reemplazando (4) en (3), el índice de polarización $\mathrm{P}_{\mathrm{EGR}}$ (o simplemente EGR) se puede escribir como:

$$
P_{E G R}=P_{E R}-\beta\left[G(f)-G\left(p^{*}\right)\right]
$$

Según Duclos et al. (2004), los índices ER y EGR poseen problemas de discontinuidad en la captura del nivel de polarización y discrecionalidad en la determinación de grupos relevantes. Por ello, los autores proponen un nuevo índice denominado DER, que constituye una extensión al índice de polarización ER. Este nuevo índice está basado en la aproximación de la 
fuerza de identificación de grupo para cada individuo. Para su cálculo se utilizan técnicas no paramétricas de kernels, mediante el valor de la función de densidad evaluada en el ingreso del individuo, tal que:

$$
P_{D E R}(\alpha, f(\cdot))=\iint f(x)^{1+\alpha} f(y)|y-x| d y d x
$$

donde $\mathrm{f}(\cdot)$ es la función de densidad de la distribución, la alienación entre dos individuos con ingresos $x$ e $y$ se va a medir a través de su distancia absoluta, y la identificación como el valor de la densidad de la distribución en el punto $\mathrm{f}(\mathrm{x})$ y $\mathrm{f}(\mathrm{y})$. El parámetro $\alpha$ es el peso asociado a la identificación dentro del índice de polarización ${ }^{1}$. Duclos et al. (2004) establecen el valor de $\alpha \in[0,25 ; 0,1]$ para que se cumpla el conjunto de axiomas que validan al índice.

\subsection{Bipolarización}

Foster y Wolfson (1992) establecen que las medidas de clase media fundamentadas en una medida de tendencia central se encuentran basadas en un rango $\mathrm{Z}=[\underline{\mathrm{z}} m, \bar{z} m]$ tal que: $\underline{\mathrm{z}} m<m<\bar{z} m$ o $0 \leq \underline{\mathrm{z}} \leq 1 \leq \bar{z}$, donde $m$ es la mediana de la distribución $F$. El tamaño de la clase media viene dado por $M(F, Z)=F(\bar{z}$ $\left.m_{F}\right)-F\left(\underline{z} m_{F}\right)$. Por lo tanto, para determinar si una distribución $F$ tiene mayor clase media que $G$, se debería comparar $M(F, Z)$ y $M(G, Z)$. No obstante, para determinar que una distribución $F$ inequívocamente presenta una clase media de mayor tamaño que una distribución $G$, es deseable que $M(F, Z) \geq M(G, Z)$ para todo $Z$ y $M(F, Z)>M(G, Z)$ para algunos $Z$.

Dado que distribuciones diferentes tendrán medianas diferentes, se requiere normalizar la distribución para la mediana $m$ derivada de $F$, tal que $\tilde{F}$ $(z)=F\left(z m_{f}\right)$, por la cual $\tilde{F}(1)=0,5$. En este sentido el indicador $M_{f}(z)$ denotará la proporción de población en $F$, cuyo ingreso recae entre $m_{f}$ y $Z m_{f}$ es decir:

$$
M_{f}(z)=|\tilde{F}(z)-\tilde{F}(1)|=\left|F\left(z m_{f}\right)-F\left(m_{f}\right)\right|
$$

No obstante, ya que $Z$ implica dos umbrales $\underline{z}$ y $\bar{z}$, el indicador $M$ puede ser expresado como:

1 Nótese que un valor de $\alpha=0$ indica que la identificación dentro de los grupos es ignorada por el índice, en este caso el índice va a ser igual al índice de Gini, mientras que ante un valor alto de $\alpha$ se da relevancia a la formación de grupos en la sociedad. 


$$
M_{\tilde{F}}(Z)=M_{\tilde{F}}(\underline{z})+M_{\tilde{F}}(\bar{z})=[\tilde{F}(1)-\tilde{F}(\underline{z})]+[\tilde{F}(\bar{z})-\tilde{F}(1)]
$$

donde $M_{\tilde{F}}(\underline{z})$ y $M_{\tilde{F}}(\bar{z})$ denotaría a la clase media baja dada por el umbral $\underline{z}$, y la clase media alta está dada por el umbral $\bar{z}$.

También desarrollan un ordenamiento parcial y curvas de bipolarización basados en la dimensión poblacional, cuya motivación es determinar la amplitud de un intervalo que contenga todos los hogares alrededor del percentil 0,5 . Se define entonces a $S(F, Q)$ como la distancia de ingreso normalizado $\tilde{y}(\bar{q})-\tilde{y}(q)$ asociado con $Q$, donde $\tilde{y}(q)=\tilde{F}^{-1}(q)$ es el ingreso normalizado por la mediana de la persona en el percentil $q$. En este sentido, el spread de ingreso se define como:

$$
S_{f}(q)=\left|\breve{F}^{-1}(q)-\breve{F}^{-1}(0,5)\right| \text { con } 0 \leq \mathrm{q} \leq 1
$$

En este caso, $\mathrm{S}$ mide la extensión o spread de un rango de ingreso asociado con $Q$; es decir, no importa cuál rango de clase media alrededor de $p=0,5$ es elegido, el spread de ingreso normalizado requerido para capturar este rango es más grande (o no más pequeño) para la distribución $G$ que para la distribución $F$. En este sentido, $G$ tiene una mayor bipolización de ingreso que $F$.

Finalmente, desarrollan una curva de polarización que denominan de segundo grado, tal que para una distribución $F$, la curva de polarización de segundo grado $B_{F}(q)=\left|\int_{q}^{0,5} S_{F}(p) d p\right|$ para todo $0 \leq q \leq 1$ es igual al área debajo de la curva de polarización de primer grado $S$ evaluada entre $0,5 \mathrm{y} q$. Esta curva considera simultáneamente las fuentes de polarización: aumento de spread y aumento de la bipolarización, por lo que acumula el spread de ingreso del centro de la distribución hacia la parte superior y la parte inferior. En tal sentido, el indicador de polarización se describe como:

$$
P_{w}=2 \frac{\mu}{m}[2(0,5-L(0,5))-G]
$$

donde $\mu$ es el ingreso medio, $m$ la mediana, $\mathrm{L}(0,5)$ el valor de la curva de Lorenz en la mediana de la distribución y $G$ el coeficiente de Gini²

2 Nótese que el índice alcanzará el valor máximo de uno cuando la mitad de la población tenga un ingreso igual a cero y la otra mitad de la población concentre dos veces la media de la distribución. 


\section{Descripción de los datos}

Las fuentes de información utilizadas para el estudio fueron las Encuestas de Empleo, Desempleo y Subempleo —Enemdu - de diciembre, entre el 2007 y el 2014, publicadas por el Instituto Nacional de Estadística y Censos - INEC-, las cuales tienen representatividad estadística a nivel nacional.

La variable utilizada para el análisis fue el ingreso per cápita familiar del hogar ${ }^{3}$. Esta variable se calcula como la razón del ingreso disponible del hogar para el número de miembros de este. Cabe señalar que, de manera general, los componentes del ingreso disponible se clasifican según fuente laboral y no laboral; por un lado, los ingresos laborales contienen los ingresos de la actividad principal y secundaria, y los ingresos no laborales se conforman por los ingresos de propiedad y transferencias. Además, se llevó a cabo el mismo proceso de depuración de los datos utilizado por el INEC (véase Castillo \& Puebla, 2016).

\section{Resultados y discusión}

Esta sección describe los resultados obtenidos en las estimaciones de clase media utilizando los enfoques de alineación-identificación para las medidas de polarización EGR y DER, así como el enfoque de bipolarización ${ }^{4}$.

\section{1. Índices de polarización EGR}

La tabla 3 muestra los resultados del cómputo del índice EGR cuando la población se divide en 2 y 3 grupos, para dos valores en $\beta$ (parámetro libre que pondera la medición del error o falta de identificación) y a su vez tres valores diferentes para $\alpha$ (parámetro del grado de sensibilidad a la identificación); además, en la última columna se presenta el índice de Gini.

Si se observan los resultados por columna, cuando la población se divide en dos grupos $(\mathrm{k}=2)$, el parámetro libre es cero $(\beta=0)$ y la sensibilidad a la identificación es uno $(\alpha=1)$, la polarización pasa de 0,41 en el 2007 a 0,34 para el 2014, es decir una reducción de 7 puntos porcentuales.

3 Para eliminar las distorsiones ocasionadas por la evolución de los precios, el ingreso fue deflactado por el índice de precios al consumidor con año base 2014 .

4 Cabe señalar que, debido a que la fuente de información utilizada proviene de encuestas, los cambios de muestreo podrían llevar a inferir conclusiones erróneas en cuanto a la evolución de los indicadores; por ello, para determinar la robustez de los resultados, el anexo A presenta un análisis de inferencia estadística en los cambios en los índices de polarización. 


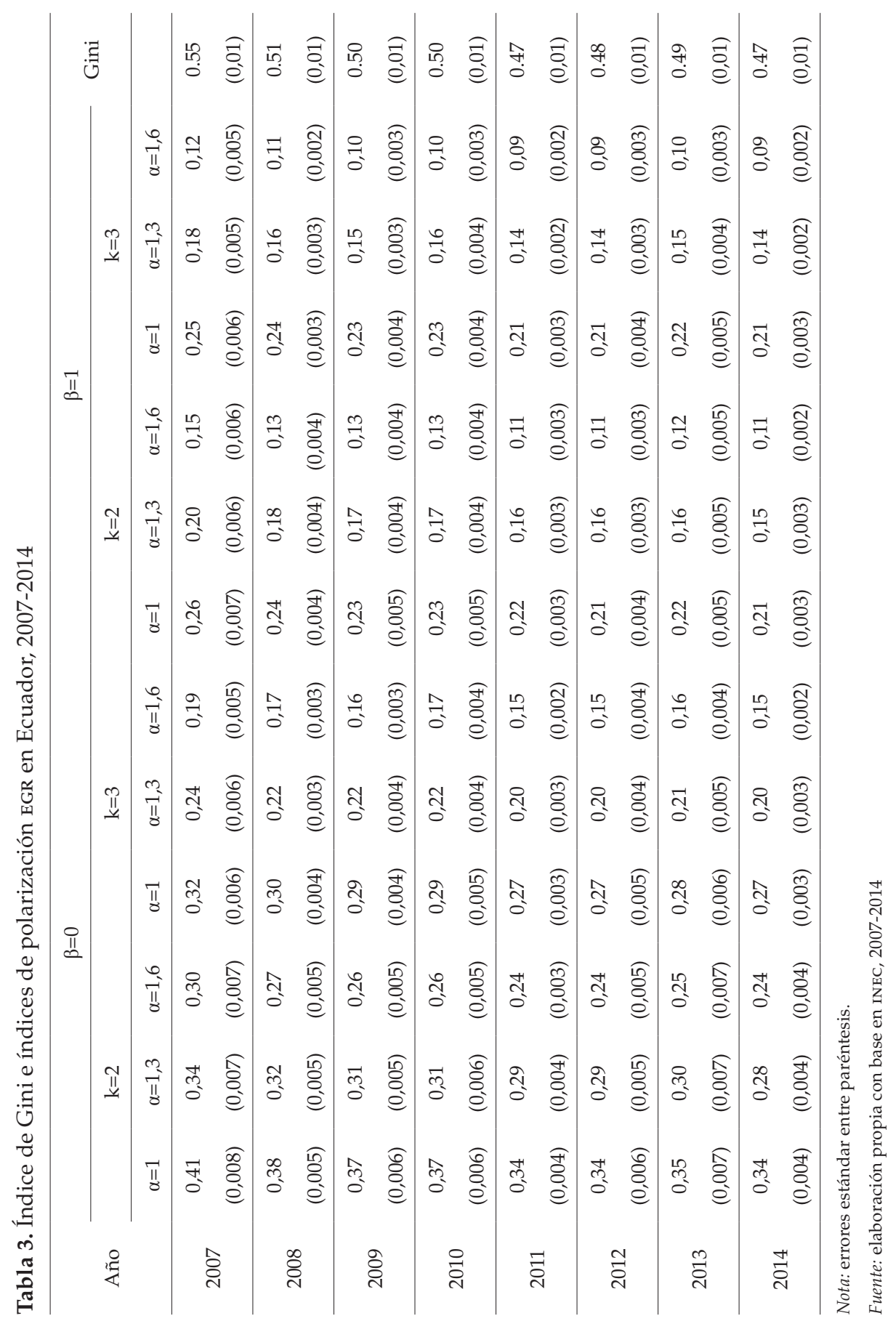


Por otro lado, los resultados por fila muestran que mientras $\alpha$ aumenta la magnitud en la variación de los índices de polarización tiende a disminuir. Además, cuando se divide en tres grupos a la población $(\mathrm{k}=3)$ se asume una división de clases en baja, media y alta. En este caso destaca que cuando los grupos $\mathrm{k}$ aumentan, la magnitud (en el índice y en su variación) para cualquier valor de $\alpha$ tiende a reducirse, pues cuando la distribución estaba dividida en dos grupos, la reducción de los índices fue de alrededor de un punto porcentual mayor para los diferentes valores de $\alpha$. De igual manera sucede al aumentar el parámetro de identificación $(\mu)$, ya que implica que los grupos están definidos de mejor manera.

En resumen, el análisis por fila nos indica que el índice EGR es altamente sensible a los parámetros que se utilicen para su estimación. Sin embargo, para los fines de esta investigación, es más interesante el análisis por columna, el cual nos muestra que independientemente de los parámetros utilizados, los índices de polarización EGR ${ }^{\alpha=1,}$ EGR $^{\alpha=1,3}$ y EGR $^{\alpha=1,6}$ durante el periodo 2007 2014, la polarización en el Ecuador disminuyó.

La última columna de la tabla 3 también muestra una disminución en términos de desigualdad, medida a través del índice de Gini. Hay que recordar que la polarización y la desigualdad miden aspectos diferentes de la distribución del ingreso, por lo tanto, son índices que se complementan dentro de un análisis. Como se mencionó, la polarización capta la formación de grupos o polos antagónicos en una distribución, mientras que la desigualdad se centra en la dispersión global de la distribución. En el caso de Ecuador, entre el 2007 y el 2014, además de disminuir la desigualdad, también disminuyó la polarización.

La tabla 4 muestra la representación de la distribución del ingreso cuando la población se divide en tres grupos ( $k=3$ : clase baja, media y alta). La tendencia a la baja de los índices de polarización y el aumento del peso relativo de la clase media muestran la mejora que este grupo ha experimentado en el periodo. Se observa que la clase media pasa del 34,58\% en el 2007 al $36,73 \%$ para el 2014 , lo que representa un aumento de 2 puntos porcentuales. También se observa una disminución del peso poblacional de la clase baja, pasando de 52,64\% en el 2007 al 48,14\% en el 2014, es decir una reducción de 5 puntos. Finalmente, la clase alta aumenta su peso en alrededor de 2 puntos porcentuales. Estas cifras infieren un desplazamiento homogéneo de los hogares de clases más bajas hacia clases más altas. Si bien es cierto que se evidencia un aumento del peso poblacional de la clase alta en el periodo de análisis, la participación del ingreso medio de este grupo reduce su peso en cuanto a la media del ingreso global en el periodo de análisis. Al contrario, la clase baja incrementa su participación con respecto al ingreso medio global 
de 0,32 en el 2007 a 0,38 en el 2014, es decir alrededor de 8 puntos durante el periodo de análisis.

Tabla 4. Índice EGR con representación óptima, para $\mathrm{k}=3$, de la distribución del ingreso per cápita familiar en Ecuador, 2007-2014 (Ingresos normalizados por la mediana global)

\begin{tabular}{ccccccc}
\hline \multirow{2}{*}{ Años } & \multicolumn{3}{c}{ Población } & \multicolumn{3}{c}{ Medias } \\
\cline { 2 - 7 } & Baja & Media & Alta & $\mu_{\text {baja }} / \mu_{\text {total }}$ & $\mu_{\text {media }} / \mu_{\text {total }}$ & $\mu_{\text {alta }} / \mu_{\text {total }}$ \\
\hline 2007 & 52,64 & 34,58 & 12,79 & 0,32 & 1,00 & 3,82 \\
2008 & 49,72 & 35,70 & 14,57 & 0,33 & 0,99 & 3,31 \\
2009 & 49,08 & 36,14 & 14,78 & 0,35 & 0,98 & 3,23 \\
2010 & 50,24 & 35,34 & 14,43 & 0,35 & 0,99 & 3,28 \\
2011 & 47,20 & 36,31 & 16,49 & 0,36 & 0,99 & 2,86 \\
2012 & 47,50 & 36,63 & 15,87 & 0,36 & 0,99 & 2,93 \\
2013 & 48,62 & 35,95 & 15,44 & 0,36 & 0,98 & 3,05 \\
2014 & 48,14 & 36,73 & 15,13 & 0,38 & 0,99 & 2,98 \\
\hline
\end{tabular}

Fuente: elaboración propia con base en INEC, 2007-2014

\section{2. Índices de polarización DER}

Para completar el análisis de la tendencia de la polarización pura del ingreso dentro del enfoque alienación-identificación, se computó también el índice DER, el cual es sensible a cambios en toda la distribución del ingreso, pues abandona el particionamiento exógeno de la distribución que da origen a un número finito y discreto de grupos de ingreso. La tabla 5 presenta los cálculos de este índice para diferentes valores del parámetro de aversión a la identificación; como se puede observar, el índice disminuye entre el 2007 y el 2014 en un promedio 4,5 puntos para todos los parámetros de aversión a la identificación $\alpha$. Al igual que para los índices EGR presentados, la magnitud de la polarización se reduce a medida que aumenta el parámetro de identificación.

Tabla 5. Polarización en Ecuador, 2007-2014. Índice DER

\begin{tabular}{ccccc}
\hline \multirow{2}{*}{ Año } & \multicolumn{5}{c}{$\alpha$} \\
\cline { 2 - 5 } & 0,25 & 0,5 & 0,75 & 1 \\
\hline \multirow{2}{*}{2007} & 0,38 & 0,31 & 0,27 & 0,25 \\
& $(0,006)$ & $(0,005)$ & $(0,006)$ & $(0,007)$ \\
\hline \multirow{2}{*}{2008} & 0,36 & 0,29 & 0,25 & 0,23 \\
& $(0,004)$ & $(0,004)$ & $(0,004)$ & $(0,005)$ \\
\hline
\end{tabular}




\begin{tabular}{ccccc}
\hline \multirow{2}{*}{ Año } & \multicolumn{4}{c}{$\alpha$} \\
\cline { 2 - 5 } & 0,25 & 0,5 & 0,75 & 1 \\
\hline \multirow{2}{*}{2009} & 0,35 & 0,28 & 0,25 & 0,22 \\
& $(0,004)$ & $(0,004)$ & $(0,004)$ & $(0,005)$ \\
\hline \multirow{2}{*}{2010} & 0,35 & 0,29 & 0,25 & 0,23 \\
& $(0,005)$ & $(0,004)$ & $(0,005)$ & $(0,005)$ \\
\hline \multirow{2}{*}{2011} & 0,34 & 0,27 & 0,23 & 0,21 \\
& $(0,003)$ & $(0,003)$ & $(0,003)$ & $(0,003)$ \\
\hline \multirow{2}{*}{2012} & 0,34 & 0,27 & 0,23 & 0,20 \\
& $(0,004)$ & $(0,004)$ & $(0,004)$ & $(0,004)$ \\
\hline \multirow{2}{*}{2013} & 0,34 & 0,28 & 0,24 & 0,22 \\
& $(0,006)$ & $(0,005)$ & $(0,005)$ & $(0,006)$ \\
\hline \multirow{2}{*}{2014} & 0,33 & 0,27 & 0,23 & 0,21 \\
& $(0,006)$ & $(0,000)$ & $(0,000)$ & $(0,000)$ \\
\hline
\end{tabular}

Nota: errores estándar entre paréntesis.

Fuente: elaboración propia con base en INEC, 2007-2014

\subsection{Análisis de bipolarización}

En esta sección se presentan los resultados del cómputo de los índices de bipolarización EGR y $\mathrm{W}^{5}$, los cuales tuvieron una disminución de 5 y 10 puntos porcentuales, respectivamente, pasando de 0,26 a 0,21 en el caso del índice EGR y de 0,52 a 0,42 en el caso del índice W. La figura 1 muestra la tendencia que tuvieron los índices en el periodo analizado.

La disminución de la bipolarización medida por estos enfoques podría estar explicada por el decrecimiento de la distancia entre los grupos. Como se observa en la figura 2, el ingreso medio del grupo de menores ingresos $(\mu 1)$ muestra una tendencia creciente a partir del 2009. Por otro lado, el grupo de mayores ingresos $(\mu 2)$ también presenta un ligero aumento en sus ingresos, pero muestra una tendencia de menor pendiente. Un indicador clave para analizar es el ratio de la media global sobre la mediana global $(\mu 2 / \mu 1)$; se puede observar que la asimetría que este ilustra desciende en el periodo de análisis.

5 Las estimaciones presentadas en el gráfico de la bipolarización se obtienen cuando $\alpha$ y $\beta$ son igual a 1 , y el corte optimo es la media. 


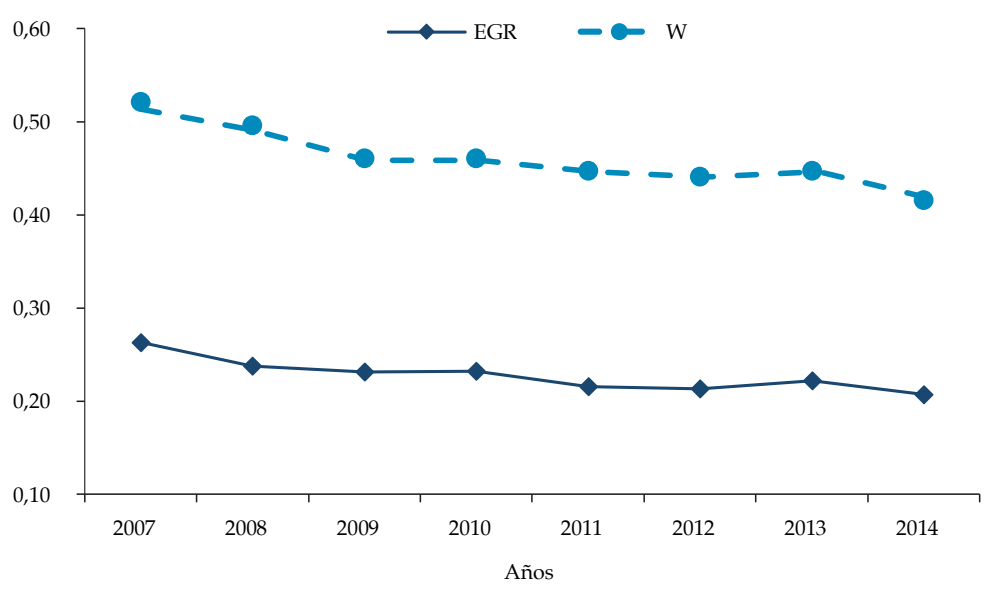

Figura 1. Bipolarización en Ecuador, 2007-2014

Fuente: elaboración propia con base en INEC, 2007-2014

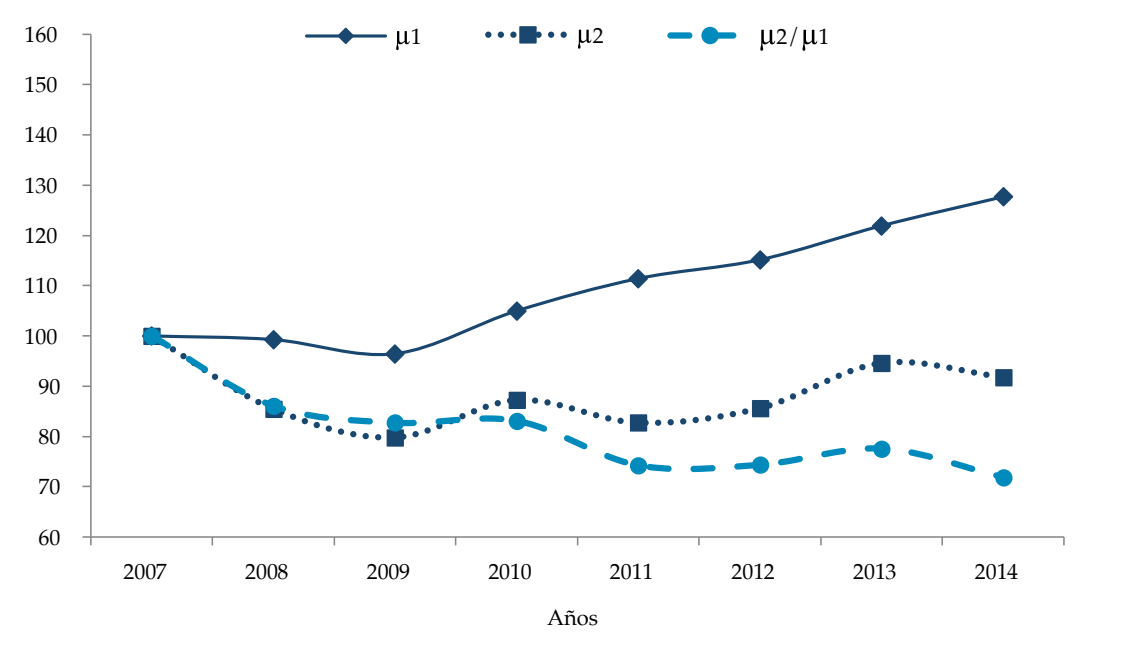

Nota: $2007=100$.

Figura 2. Incremento entre grupos por encima y por debajo del ingreso mediano en Ecuador, 2007-2014

Fuente: elaboración de los autores con base en INEC, 2007-2014

Siguiendo a Foster y Wolfson (1992), la tabla 6 presenta ciertos elementos estadísticos que ayudan a explicar las curvas desarrolladas por los autores. La primera sección de indicadores se refiere a la proporción poblacional dentro de un rango específico de ingresos. Así, por ejemplo, se observa que alrededor del $12 \%$ de personas tiene un ingreso menor al $40 \%$ de la mediana para el 2007; esta proporción disminuye al $10 \%$ para el 2014. Es importante 
notar que para porcentajes bajos de la mediana, la proporción poblacional ha disminuido; no obstante, a partir del rango del $60 \%$ al $75 \%$ la proporción poblacional tiende a aumentar ligeramente. Este comportamiento nos permite inferir un aumento de la clase media entre el 2007 y el 2014.

Esta percepción puede ser confirmada al usar la curva M. Bajo este enfoque, la clase media se ha incrementado en alrededor de 4 puntos porcentuales entre el 2007 y el 2014. De igual manera, al analizar los rangos poblacionales alrededor de la media, se puede observar que se requiere un spread del ingreso menor para capturar los diferentes rangos poblacionales hacia el 2014, lo que refleja una menor variación del ingreso en la distribución para este año. Así, por ejemplo, dado un rango poblacional del $20 \%$ al $80 \%$, en el 2007 , se requiere un spread del ingreso del $24 \%$ de la mediana; este porcentaje decrece en 3,7\% para el 2014.

Tabla 6. Elementos de la bipolarización, Ecuador 2007-2014

\begin{tabular}{|c|c|c|c|}
\hline & 2007 & 2014 & Diferencia \\
\hline \multicolumn{4}{|c|}{ \% población con ingresos } \\
\hline$<40 \%$ de la mediana & 12,41 & 10,96 & $-1,46$ \\
\hline$<50 \%$ de la mediana & 17,25 & 16,85 & $-0,4$ \\
\hline$<60 \%$ de la mediana & 22,93 & 22,92 & $-0,01$ \\
\hline $60 \%$ a $75 \%$ & 8,38 & 9,42 & 1,04 \\
\hline $75 \%$ a $100 \%$ & 12,19 & 13,98 & 1,79 \\
\hline $200 \%$ a $125 \%$ & 9,65 & 11,64 & 1,99 \\
\hline $125 \%$ a $150 \%$ & 7,59 & 8,14 & 0,55 \\
\hline $200 \%$ & 70,97 & 77,99 & 7,03 \\
\hline \multicolumn{4}{|c|}{$\%$ en la curva $\mathrm{M}$ dado un rango de ingreso } \\
\hline $75 \%$ a $150 \%$ mediana & 29,42 & 33,62 & 4,20 \\
\hline $75 \%$ a $125 \%$ & 21,83 & 25,48 & 3,65 \\
\hline $50 \%$ a $150 \%$ & 43,48 & 49,10 & 5,62 \\
\hline \multicolumn{4}{|c|}{ Spread dado en rango de población } \\
\hline $40 \%$ a $60 \%$ & 2,52 & 2,12 & $-0,4$ \\
\hline $35 \%$ a $65 \%$ & 5,59 & 4,78 & $-0,81$ \\
\hline $30 \%$ a $70 \%$ & 10,1 & 8,59 & $-1,52$ \\
\hline $25 \%$ a $75 \%$ & 15,75 & 13,67 & $-2,08$ \\
\hline $20 \%$ a $80 \%$ & 23,93 & 20,25 & $-3,68$ \\
\hline
\end{tabular}




\begin{tabular}{|c|c|c|c|}
\hline & 2007 & 2014 & Diferencia \\
\hline \multicolumn{4}{|c|}{ Distancia promedio dado un rango de población } \\
\hline $40 \%$ a $60 \%$ & 0,12 & 0,10 & $-0,02$ \\
\hline $35 \%$ a $65 \%$ & 0,18 & 0,15 & $-0,03$ \\
\hline $30 \%$ a $70 \%$ & 0,25 & 0,21 & $-0,04$ \\
\hline $25 \%$ a $75 \%$ & 0,32 & 0,27 & $-0,05$ \\
\hline $20 \%$ a $80 \%$ & 0,40 & 0,33 & $-0,07$ \\
\hline
\end{tabular}

Fuente: elaboración propia con base en INEC, 2007-2014

La figura 3 ilustra todas las características observadas en la tabla 6. En el panel superior se encuentra graficada la curva M o curva de clase media, la cual mide la concentración de las masas alrededor de la mediana de la distribución del ingreso. Se puede observar que la curva $\mathrm{M}$ de la distribución del ingreso del 2014 se encuentra sobre la curva M de la distribución del ingreso del 2007 (y no se cruzan), por lo que la proposición 1 se cumple: la distribución del ingreso del 2014 tiene una clase media más grande que la distribución del ingreso en el 2007; es decir, la distribución del ingreso del 2014 concentra mayor masa alrededor de la mediana que la distribución del ingreso del 2007.

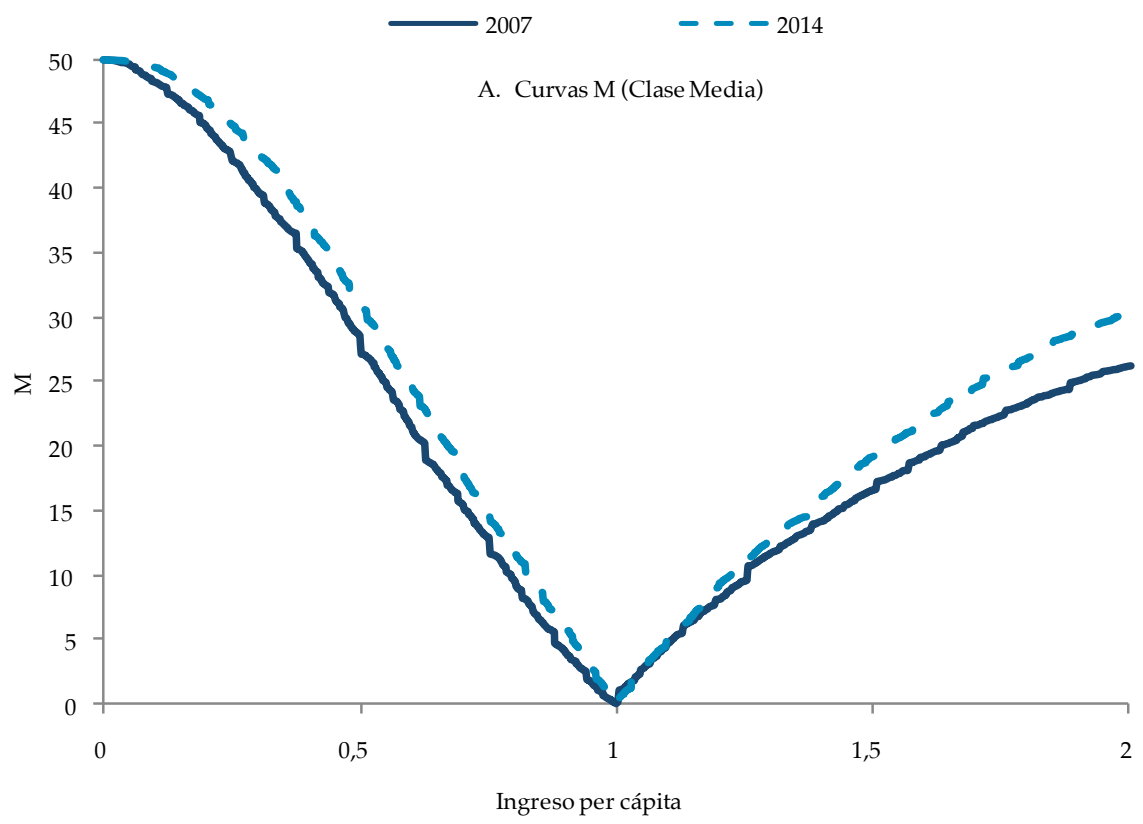



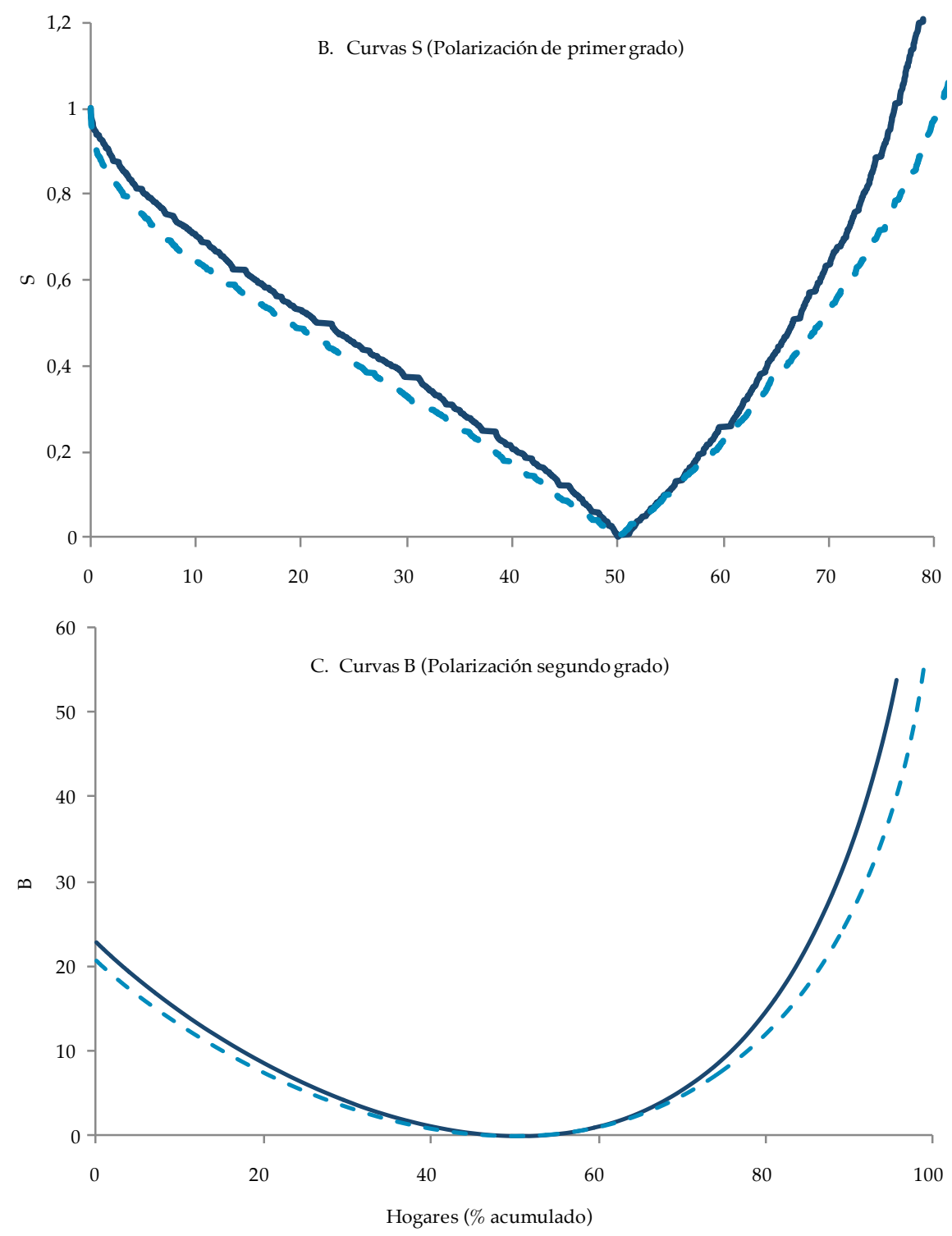

Figura 3. Curvas de clase media y polarización, Ecuador 2007-2014 Fuente: elaboración propia con base en INEC, 2007-2014

En el mismo sentido, las curvas de polarización de primer y segundo grado (panel medio e inferior) indican que la polarización en la distribución del ingreso en el 2007 es mayor que la polarización en la distribución del ingreso en el 2014, revelando que la primera presenta mayor spread del ingreso. En este sentido, un mayor spread del ingreso implica una menor proporción de 
población alrededor de la mediana y, según la proposición 2, la distribución del ingreso del 2014 presenta una mayor clase media que la distribución del ingreso del 2014. Como se observa, la curva S del 2007 domina a la curva del 2014. De igual manera, al observar la gráfica de las curvas de polarización de segundo grado, la curva del 2014 se encuentra debajo de la curva del 2007, lo que implica que la distribución del ingreso en el 2007 presenta un mayor spread del ingreso, al igual que mayor bipolaridad que la distribución del ingreso en el 2014.

En general, tras el análisis del conjunto de indicadores de polarización y bipolarización, queda claro que durante el periodo 2007-2014 existe una mejora distributiva a nivel nacional, que no solo disminuyó los niveles de desigualdad sino que también redujo los niveles de polarización del ingreso, lo cual se ve reflejado tanto en la tendencia a la baja de los indicadores de polarización del ingreso, como en el aumento en términos de participación poblacional y de ingreso medio del grupo con ingresos bajos y medios, produciendo un incremento de la clase media en el Ecuador.

Si bien el análisis realizado en este documento no pretende ser un análisis causal, es importante mencionar factores, tanto de orden económico como de políticas públicas, que pudieron haber influido en los resultados presentados. Así, recientes investigaciones del Banco Mundial (2014), Cruces et al. (2015) y Cuevas, Atuesta y Zanbonino (2016) determinan que los factores clave que contribuyeron al proceso de redistribución del ingreso durante el periodo de análisis fueron el ingreso laboral y las transferencias públicas.

Según Cuevas et al. (2016), el ingreso laboral se incrementó en todos los grupos socioeconómicos, pero más entre los percentiles más bajos de la distribución. El ingreso laboral per cápita promedio en las áreas urbanas aumentó en $25 \%$ entre el 2006 y el 2014, mientras que para el primer y segundo decires de la distribución ( $20 \%$ de la población) subió en $41 \%$ y 51 \%. Es importante mencionar que el panorama en cuanto al ingreso laboral se debe principalmente a políticas de inversión pública y legislación en el mercado laboral, entre las que destacan: 1) inversión pública en construcción y la metalurgia; 2) ley y afiliación de la seguridad social; 3) incremento constante del salario básico unificado $3 / 4 \mathrm{SBU} 3 / 4$ que fue elevado de US\$ 170 al mes en el 2007 a US\$ 340 al mes en el 2014.

Queda claro, entonces, que varias de las políticas adoptadas con fines redistributivos influyeron en una mejora de la equidad y en un aumento de la clase media en el país. Sin embargo, queda todavía por determinar si los cambios obtenidos son estructurales y sostenibles en el tiempo, ya que ante el último incremento del desempleo o empleo inadecuado podrían verse afectados muchos de los logros alcanzados. 


\section{Conclusiones}

La expansión de la clase media es una de las cuestiones claves que contribuyen a la reducción de la desigualdad y la polarización en una sociedad. No obstante, para medir y monitorear a este grupo se presenta la dificultad de la falta de un consenso en cuanto a su definición operacional. La determinación del enfoque a utilizar depende de los objetivos de la investigación. En este trabajo, las definiciones basadas en medidas endógenas asociadas a los conceptos de polarización y bipolarización resultan las más adecuadas, ya que reducen la arbitrariedad de las definiciones tradicionales basadas en percentiles y líneas de pobreza.

La polarización económica se encuentra relacionada con el tamaño de la clase media, ya que el fenómeno de la polarización puede ser entendido como la formación de grupos con ingresos homogéneos en su interior, pero que, a la vez, presentan una fuerte heterogeneidad respecto de los otros grupos. En este sentido, un debilitamiento de los grupos de ingreso medio a costa de un aumento de los grupos extremos ricos y pobres tendería a incrementar la polarización.

Es importante considerar que los índices de polarización y bipolarización son altamente sensibles al cambio en los parámetros que se utilizan para sus estimaciones. En ese sentido, destacan el número de grupos en que se dividirá a la población y el parámetro de sensibilidad a la identificación como factores relevantes para la estimación de los índices.

Diversos estudios han demostrado las mejoras que ha conseguido el Ecuador en términos de desigualdad y equidad en los últimos años. El presente estudio complementa dichos análisis y demuestra que, además de mejoras en términos de desigualdad e inequidad, el país también consiguió mejoras en términos de polarización.

Los resultados obtenidos dan cuenta de que la cohesión social en el Ecuador, medida a través de los índices de polarización o bipolarización, disminuyó independientemente del índice o de los parámetros que se utilicen para su estimación. Además, destaca el desplazamiento homogéneo de los hogares de clases más bajas hacia clases más altas, lo que demuestra que la clase media, cuantificada como proporción de la población total, se incrementó durante dicho periodo.

El incremento de la clase media y la disminución de la polarización tendrían su explicación en la mejora de la distribución del ingreso a nivel nacional. Esto se ve reflejado tanto en la tendencia a la baja de los indicadores de polarización, como en el aumento en términos de participación poblacional y de ingreso medio del grupo con ingresos bajos y medios. La evidencia sobre 
las mejoras en la distribución del ingreso y, por ende, en la disminución de la desigualdad son corroboradas en varios estudios realizados para el caso ecuatoriano, en el que destacan políticas de inversión pública y legislación en el mercado laboral para alcanzar dichos resultados.

Finalmente, se plantea como una línea de investigación para superar limitaciones que tiene el estudio, en particular referente a las fuentes de información, la utilización de bases de datos alternas a encuestas de hogares, por ejemplo, registros administrativos de instituciones públicas que recojan información de ingresos y características socioeconómicas de la población, como los registros administrativos del Servicio de Rentas Internas y el Instituto Ecuatoriano de Seguridad Social. Este ejercicio podría enriquecer el análisis ya que deja de lado ciertas desventajas del uso de encuestas, como el problema de la subdeclaración de ingresos y la representatividad estadística en territorios geográficos de mayor desagregación.

\section{Referencias}

Acemoglu, D. \& Zilibotti, F. (1997). Was Prometheus unbound by chance? Risk, diversification and growth. The Journal of Political Economy, 105(4), 709-751.

Banco Mundial (2014). Ecuador: informalidad y mercado laboral. Informe 90486-EC. Quito.

Banerjee, A. \& Duflo, E. (2008). What Is middle class about the middle classes around the world? Journal of Economic Perspectives, 22(2), 3-28.

Blackburn, M. \& Bloom, D. (1985). What is happening to the middle class? American Demographics, 7(1), 19-25.

Borraz, F., González, N. \& Rossi, M. (2013). Polarization and the middle class in Uruguay. Latin American Journal of Economics, 50(2), 289-326. Dor: 10.7764.

Castillo, R. \& Puebla, D. (2016). Aspectos metodológicos sobre la medición de la pobreza por ingresos en el Ecuador. Revista de Estadística y Metodologías, 2(1),7-26.

Cruces, G., Fields, G., Jaume, D. \& Viollaz, M. (2015). The growth-employmentpoverty nexus in Latin America in the 2000s: Ecuador country study. ,Wider Working Paper 2015/076. Helsinski: unu-Wider.

Cruces, G., López-Calva, L. \& Battiston, D. (2010). Down and out or up and in? In search of Latin America's elusive middle class. Research for public policy, inclusive development, ID-03-2010, RBLAC-UNDP. Nueva York: United Nations Development Program. 
Cuevas, F., Atuesta, B. \& Zanbonino, D. (2016). ¿Qué impulsó la reducción de la pobreza y la desigualdad en Ecuador en la década pasada? Una historia del mercado laboral urbano. En Reporte de pobreza por consumo Ecuador 2006-2014 (pp. 210-259). Quito: InEc/Banco Mundial.

D'Ambrosio, C., Muliere, P. \& Secchi, P. (2002). Income thresholds and income classes. Discussion Papers of DIW Berlin 325. Berlín: German Institute for Economic Research.

Doepke, M. \& Zilibotti, F. (2005). Social class and the spirit of capitalism. Journal of the European Economic Association, 3(2-3), 516-524.

Duclos, J.-Y., Esteban, J. \& Ray, D. (2004). Polarization: concepts, measurement, estimation. Econometrica. Journal of the Econometric Society, 72(6), 1737-1772.

Edo, M. \& Sosa-Escudero, W. (2013). Assessing the measurement of the middle class: Argentina 1991-2012. Ponencia presentada en la Conferencia ECINEQ, Bari, Italia.

Esteban, J. M. \& Ray, D. (1994). On the measurement of polarization. Econometrica: Journal of the Econometric Society, 62(4), 819-851.

Esteban, J., Gradín, C. \& Ray, D. (2007). An extension of a measure of polarization, with an application to the income distribution of five OECD countries. The Journal of Economic Inequality, 5(1), 1-19.

Ferreira, F., Messina, J., Rigolini, J., López-Calva, L., Lugo, M. \& Vakis, R. (2013). La movilidad económica y el crecimiento de la clase media en América Latina. Washington, DC: Banco Mundial.

Foster, J. \& Wolfson, M. (1992). Polarization and the decline of the middle class: Canada and the US. OPHI Working Paper 31. Oxford Poverty and Human Development Initiative.

Gasparini, L., Horenstein, M. \& Olivieri, S. (2006). Economic polarization in Latin America and the Caribbean: what do household surveys tell us? Cedlas Working Papers 0038., La Plata: Cedlas/Universidad Nacional de La Plata. Gasparini, L., Horenstein, M., Molina, E. \& Olivieri, S. (2008). Income polarization in Latin America: patterns and links with institutions and conflict. Oxford Development Studies, 36(4): 463-484.

Gradín, C. (2000). Polarization by sub-populations in Spain. The Review of Income and Wealth, 46(4), 457-474.

Gradín, C. \& Rossi, M. (2000). Polarization and wage inequality in Uruguay, 1989-1997. El Trimestre Económico, 67(267), 421-443.

Huesca, L. (2003). Análisis de polarización del ingreso de los hogares en México durante 1984-2000. Investigación Económica, LXII(246), 89-123.

Instituto Nacional de Estadística y Censos -INEC-. 2007-2014. Encuesta Nacional de Empleo, Desempleo y Subempleo (Enemdu). Recuperado 
de http:/ / www.ecuadorencifras.gob.ec/empleo-encuesta-nacional-deempleo-desempleo-y-subempleo-enemdu /

Kharas, H. (2010). The emerging middle class in developing countries. Working Paper . $^{\circ}$ 285. Development Centre, OECD.

Larrú, J. M. (2013). Polarización, desigualdad y ayuda al desarrollo en América Latina. América Latina Hoy, (63), 45-64.

López-Calva, L. F. \& Ortiz-Juarez, E. (2012). Clases medias y vulnerabilidad a la pobreza en América Latina. Pensamiento Iberoamericano, (10), 49-70. López-Calva, L. F. \& Ortiz-Juarez, E. (2013). A vulnerability approach to the definition of the middle class. The Journal of Economic Inequality, 12(1), 23-47. Dor: 10.1007 /s10888-012-9240-5

Massari, R., Pittau, M. \& Zelli, R. (2009). A dwindling middle class? Italian evidence in the 2000s. The Journal of Economic Inequality, 7(4), 333-350.

Nayab, D. (2011). Estimating the middle class in Pakistan. The Pakistan Development Review, 1(50), 1-28.

Olivieri, S. (2007). Debilitamiento de la clase media: Gran Buenos Aires 1986-2004. La Plata: Universidad Nacional de La Plata.

Pendfold, M. \& Rodríguez, G. (2014). La creciente pero vulnerable clase media de América Latina: patrones de expansión, valores y preferencias. En Serie políticas públicas y transformación productiva $n .^{\circ} 17$. Corporación Andina de Fomento - CAF-.

Reynal-Querol, M. (2002). Ethnicity, political system and civil wars. Journal of Conflict Resolution, 46(1), 29-54.

Solimano, A. (2008). The middle class and the development process. Serie Macroeconomía del Desarrollo n. $^{\circ}$ 65. Santiago de Chile: Comisión Económica para América Latina y el Caribe.

Thurow, L. C. (1984). The disappearance of the middle class. The New York Times. Recuperado de https:/ / www.nytimes.com/1984/02/05/business / business-forum-the-disappearance-of-the-middle-class.html

Viollaz, M. (2008). Polarización de ingresos laborales: Argentina 1992-2006. Documento de Trabajo n. $^{\circ}$ 70. La Plata: Centro de Estudios Distributivos, Laborales y Sociales, Universidad de la Plata.

Wolfson, M. (1994). When inequalities diverge. American Economic Review Papers and Proceedings, 84(2), 353-358.

Zhang, X. \& Kanbur, R. (2001). What difference do polarization measures make? An application to China. Journal of Development Studies, (37), 85-98.

Zhu, F. (2005). A nonparametric analysis of the shape dynamics of the us personal income distribution: 1962-2000. BIS Working paper $n .{ }^{\circ} 184$. Washington, DC: Bank for International Settlements, Monetary and Economic Department. 


\section{Anexos}

Anexo A. Análisis de robustez estadística: inferencia sobre los cambios en la polarización y la clase media

Debido a que los cambios de muestreo de las encuestas podrían llevar a inferir conclusiones erróneas en cuanto a la evolución de los indicadores de polarización, se realiza un análisis de inferencia estadística en los cambios en los índices de polarización presentados en el documento. Los intervalos de confianza utilizados se construyeron al 95\% de confianza mediante la técnica de boostrap. En este sentido, se prueba la hipótesis de un aumento significativo de los índices de polarización cuando los cortes de ingreso son endógenos, es decir, la distribución boostrap $\mathrm{d}=\mathrm{s}_{1}-\mathrm{s}_{2}=0$ contra la hipótesis alternativa $\mathrm{d}>0$, donde $\mathrm{s}_{1} \mathrm{y} \mathrm{s}_{2}$ son los limites de los intervalos de confianza.

La tabla 7 presenta, además de las estimaciones de los índices de bipolarización (EGR, W), sus intervalos de confianza, los cuales fueron analizados para la construcción de las tablas 8 y 9. Para establecer una disminución estadísticamente significativa de los índices de polarización, el límite inferior del año de referencia debe ser más alto que el límite superior del año a comparar. Lo anterior es indispensable ya que, como señala Huesca (2003, p. 115), "de otra manera no se podrá concluir nada al respecto, dado que, la hipótesis de igualdad entre los índices no puede ser rechazada debido a que los intervalos de confianza de los índices se solapan".

Tabla 7. Intervalos de confianza, bipolarización en Ecuador 2007-2014

\begin{tabular}{|c|c|c|c|c|c|c|c|c|c|c|c|c|}
\hline \multirow{2}{*}{$\begin{array}{l}\text { 足 } \\
\text { 安 }\end{array}$} & \multicolumn{3}{|c|}{$\alpha=1$} & \multicolumn{3}{|c|}{$\alpha=1,3$} & \multicolumn{3}{|c|}{$\alpha=1,6$} & \multirow[b]{2}{*}{$\begin{array}{l}\text { Estima- } \\
\text { ción }\end{array}$} & \multirow[b]{2}{*}{$x_{0}$} & \multirow[b]{2}{*}{ LS } \\
\hline & $\begin{array}{l}\text { Estima- } \\
\text { ción }\end{array}$ & LI & LS & $\begin{array}{l}\text { Estima- } \\
\text { ción }\end{array}$ & LI & LS & $\begin{array}{l}\text { Estima- } \\
\text { ción }\end{array}$ & LI & LS & & & \\
\hline 2007 & 0,26 & 0,250 & 0,276 & 0,20 & 0,189 & 0,212 & 0,15 & 0,141 & 0,164 & 0,52 & 0,497 & 0,545 \\
\hline 2008 & 0,24 & 0,230 & 0,246 & 0,18 & 0,170 & 0,185 & 0,13 & 0,123 & 0,137 & 0,50 & 0,475 & 0,516 \\
\hline 2009 & 0,23 & 0,222 & 0,240 & 0,17 & 0,164 & 0,180 & 0,13 & 0,118 & 0,133 & 0,46 & 0,443 & 0,478 \\
\hline 2010 & 0,23 & 0,223 & 0,241 & 0,17 & 0,165 & 0,181 & 0,13 & 0,119 & 0,135 & 0,46 & 0,443 & 0,477 \\
\hline 2011 & 0,22 & 0,209 & 0,222 & 0,16 & 0,153 & 0,164 & 0,11 & 0,108 & 0,119 & 0,45 & 0,430 & 0,463 \\
\hline 2012 & 0,21 & 0,205 & 0,221 & 0,16 & 0,149 & 0,162 & 0,11 & 0,104 & 0,116 & 0,44 & 0,422 & 0,459 \\
\hline 2013 & 0,22 & 0,211 & 0,232 & 0,16 & 0,155 & 0,174 & 0,12 & 0,110 & 0,129 & 0,45 & 0,429 & 0,465 \\
\hline 2014 & 0,21 & 0,201 & 0,213 & 0,15 & 0,146 & 0,157 & 0,11 & 0,103 & 0,113 & 0,42 & 0,402 & 0,428 \\
\hline
\end{tabular}

Nota: intervalos de confianza estimados mediante boostrap, 100 replicaciones.

Fuente: elaboración de los autores con base en INEc, 2007-2014 
Tabla 8. Cambios en los índices de bipolarización en Ecuador, 2007-2014 (índice egr, $\mathrm{K}=2$ )

\begin{tabular}{lcccccccc}
\hline Años & 2007 & 2008 & 2009 & 2010 & 2011 & 2012 & 2013 & 2014 \\
\hline 2007 & - & & & & & & & \\
2008 & Superior & - & & & & & & \\
2009 & Superior & NS & - & & & & \\
2010 & Superior & NS & NS & - & & & \\
2011 & Superior & Superior & NS & NS & - & & \\
2012 & Superior & Superior & NS & NS & NS & - & \\
2013 & Superior & NS & NS & NS & NS & NS & - & \\
2014 & Superior & Superior & Superior & Superior & NS & NS & NS & - \\
\hline
\end{tabular}

Los cambios significativos en los límites superior e inferior se toman al menos por medio punto porcentual. NS: diferencia no significativa

Fuente: elaboración de los autores con base en la tabla 7, $\alpha=1$

Con respecto al índice EGR cuando $\mathrm{k}=2$, la tabla 8 muestra claramente que la bipolarización disminuye durante el periodo analizado, siendo más alto el índice en el 2007 con respecto a todos los demás años. Sin embargo, se puede notar que, conforme el año de referencia aumenta, las variaciones significativamente estadísticas tienden a reducirse. Se puede concluir que a partir del 2011 la bipolarización medida por el índice EGR $(k=2)$ es estadísticamente igual al comparar el índice con años posteriores.

Por su parte, el índice W (tabla 9) muestra características similares al índice anterior. Se puede observar también que la bipolarización medida por el índice $\mathrm{W}$ disminuye durante el periodo analizado. El índice registrado en el 2007 es el más alto con respecto a los demás (excepto contra el 2008). De igual manera, conforme el año de referencia aumenta, las variaciones significativamente estadísticas tienden a reducirse. Para este caso se concluye que desde el 2012 la bipolarización medida por W se mantiene estadísticamente igual al comparar con el 2013 y el 2014.

Tabla 9. Cambios en los indices de bipolarización en Ecuador (índice W)

\begin{tabular}{ccccccccc}
\hline Años & 2007 & 2008 & 2009 & 2010 & 2011 & 2012 & 2013 & 2014 \\
\hline 2007 & - & & & & & & & \\
2008 & NS & - & & & & & & \\
2009 & Superior & NS & - & & & & & \\
\hline
\end{tabular}




\begin{tabular}{ccccccccc}
\hline Años & 2007 & 2008 & 2009 & 2010 & 2011 & 2012 & 2013 & 2014 \\
\hline 2010 & Superior & NS & NS & - & & & \\
2011 & Superior & Superior & NS & NS & - & & \\
2012 & Superior & Superior & NS & NS & NS & - & & \\
2013 & Superior & Superior & NS & NS & NS & NS & - & \\
2014 & Superior & Superior & Superior & Superior & Superior & NS & NS & - \\
\hline
\end{tabular}

Los cambios significativos en los límites superior e inferior se toman al menos por medio punto porcentual. NS: diferencia no significativa.

Fuente: elaboración de los autores con base en la tabla 7.

Las tablas 10 y 11 muestran la réplica del ejercicio anterior para el índice EGR, ahora con $\mathrm{k}=3$. Al igual que los índices anteriores, se observa claramente que la disminución en el índice de polarización es estadísticamente significativa, puesto que el 2007 presenta los niveles de polarización más altos al comprarlos contra los años siguientes a partir del $2009^{6}$. Se puede concluir que a partir del 2011 no ha existido una variación estadísticamente significativa hasta el 2014.

Tabla 10. Intervalos de confiaza, polarización en Ecuador 2007-2014 (índice egr, k = 3)

\begin{tabular}{ccccccccccc}
\hline \multirow{2}{*}{ Años } & \multicolumn{3}{c}{$\alpha=1$} & \multicolumn{3}{c}{$\alpha=1,3$} \\
\cline { 2 - 10 } & Estimación & LI & LS & Estimación & LI & LS & Estimación & LI & LS \\
\hline 2007 & 0,25 & 0,244 & 0,266 & 0,18 & 0,167 & 0,187 & 0,12 & 0,112 & 0,130 \\
2008 & 0,24 & 0,228 & 0,242 & 0,16 & 0,155 & 0,166 & 0,11 & 0,102 & 0,111 \\
2009 & 0,23 & 0,220 & 0,234 & 0,15 & 0,148 & 0,159 & 0,10 & 0,096 & 0,106 \\
2010 & 0,23 & 0,221 & 0,238 & 0,16 & 0,149 & 0,164 & 0,10 & 0,097 & 0,111 \\
2011 & 0,21 & 0,209 & 0,219 & 0,14 & 0,139 & 0,147 & 0,09 & 0,089 & 0,096 \\
2012 & 0,21 & 0,207 & 0,221 & 0,14 & 0,137 & 0,149 & 0,09 & 0,087 & 0,098 \\
2013 & 0,22 & 0,210 & 0,228 & 0,15 & 0,140 & 0,155 & 0,10 & 0,090 & 0,103 \\
2014 & 0,21 & 0,203 & 0,214 & 0,14 & 0,136 & 0,144 & 0,09 & 0,087 & 0,095 \\
\hline
\end{tabular}

Nota: intervalos de confianza estimados mediante boostrap, 100 replicaciones.

Fuente: elaboración de los autores con base en INEc, 2007-2014

6 En ek 2008 la variación no es estadísticamente significativa. 
Tabla 11. Cambios en los indices de polarización (índice egr, k=3)

\begin{tabular}{lcccccccc}
\hline Años & 2007 & 2008 & 2009 & 2010 & 2011 & 2012 & 2013 & 2014 \\
\hline 2007 & - & & & & & & & \\
2008 & ns & - & & & & & & \\
2009 & Mayor & ns & - & & & & \\
2010 & Mayor & ns & NS & - & & & \\
2011 & Mayor & Mayor & ns & Ns & - & & \\
2012 & Mayor & ns & ns & ns & Ns & - & \\
2013 & Mayor & ns & ns & ns & ns & Ns & - & \\
2014 & Mayor & Mayor & Mayor & Mayor & ns & ns & NS & - \\
\hline
\end{tabular}

Los cambios significativos en los límites superior e inferior se toman al menos por medio punto porcentual. NS: diferencia no significativa.

Fuente: elaboración de los autores con base en la tabla 10, $\alpha=1$

Adicionalmente, se analizó el índice DER. Las tablas 12 y 13 muestran la réplica para este. Se puede observar que, a diferencia de los índices observados, el índice DER parece presentar menor variabilidad estadísticamente significativa, pues entre el 2007 y el 2009 la variación no es significativa. Al igual que los otros índices, conforme aumenta el año de referencia para el análisis, las variaciones tienden a no ser significativas. Para este caso, se puede concluir que a partir del 2011 la polarización medida por el DER no ha variado en comparación a años posteriores.

De manera global se observa que entre el 2007 y el 2014 las variaciones negativas son estadísticamente significativas para todos los índices de polarización, por lo que se podría inferir que la reducción es inequívoca. No obstante, a partir del 2009 estas variaciones tienden a ser netamente muéstrales; como se observó, para la mayoría de los casos a partir del 2011 los índices no han variado de manera estadísticamente significativa, lo que permite concluir que el periodo real de reducción continua en polarización se da entre el 2007 y el 2011. ${ }^{7}$

Con respecto al incremento proporcional de la clase media, para probar la significancia estadística entre proporciones, se utilizaron regresiones simples entre la variable dicotómica de clase media y los años a comparar, tomando

7 Es importante destacar que durante todo el periodo de análisis y para todos los índices presentados no se registra un aumento estadísticamente significativo en la polarización. 
Tabla 12. Intervalos de confiaza, polarización en Ecuador 2007-2014 (índice der)

\begin{tabular}{|c|c|c|c|c|c|c|c|c|c|c|c|c|}
\hline \multirow{2}{*}{ 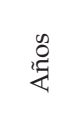 } & \multicolumn{3}{|c|}{$\alpha=0,25$} & \multicolumn{3}{|c|}{$\alpha=0,50$} & \multicolumn{3}{|c|}{$\alpha=0,75$} & \multicolumn{3}{|c|}{$\alpha=1$} \\
\hline & $\begin{array}{c}\text { Esti- } \\
\text { mación }\end{array}$ & LI & LS & $\begin{array}{c}\text { Esti- } \\
\text { mación }\end{array}$ & LI & LS & $\begin{array}{c}\text { Esti- } \\
\text { mación }\end{array}$ & LI & LS & $\begin{array}{c}\text { Esti- } \\
\text { mación }\end{array}$ & LI & LS \\
\hline 2007 & 0,38 & 0,370 & 0,391 & 0,31 & 0,299 & 0,319 & 0,27 & 0,262 & 0,285 & 0,25 & 0,240 & 0,267 \\
\hline 2008 & 0,36 & 0,351 & 0,368 & 0,29 & 0,282 & 0,297 & 0,25 & 0,243 & 0,260 & 0,23 & 0,219 & 0,238 \\
\hline 2009 & 0,35 & 0,344 & 0,362 & 0,28 & 0,276 & 0,292 & 0,25 & 0,238 & 0,255 & 0,22 & 0,214 & 0,233 \\
\hline 2010 & 0,35 & 0,345 & 0,364 & 0,29 & 0,278 & 0,295 & 0,25 & 0,241 & 0,259 & 0,23 & 0,218 & 0,239 \\
\hline 2011 & 0,34 & 0,330 & 0,343 & 0,27 & 0,265 & 0,275 & 0,23 & 0,226 & 0,237 & 0,21 & 0,201 & 0,214 \\
\hline 2012 & 0,34 & 0,329 & 0,346 & 0,27 & 0,262 & 0,277 & 0,23 & 0,222 & 0,238 & 0,20 & 0,196 & 0,213 \\
\hline 2013 & 0,34 & 0,331 & 0,355 & 0,28 & 0,268 & 0,288 & 0,24 & 0,231 & 0,253 & 0,22 & 0,208 & 0,232 \\
\hline 2014 & 0,33 & 0,323 & 0,337 & 0,27 & 0,261 & 0,272 & 0,23 & 0,225 & 0,237 & 0,21 & 0,201 & 0,215 \\
\hline
\end{tabular}

Nota: intervalos de confianza estimados mediante boostrap, 100 replicaciones.

Fuente: elaboración de los autores con base en INEC, 2007-2014

Tabla 13. Cambios en los índices de polarización (índice der)

\begin{tabular}{lcccccccc}
\hline Años & 2007 & 2008 & 2009 & 2010 & 2011 & 2012 & 2013 & 2014 \\
\hline 2007 & - & & & & & & & \\
2008 & NS & - & & & & & & \\
2009 & NS & NS & - & & & & & \\
2010 & NS & NS & NS & - & & & & \\
2011 & Superior & Superior & NS & NS & - & & \\
2012 & Superior & Superior & NS & NS & NS & - & & \\
2013 & Superior & NS & NS & NS & NS & NS & - & \\
2014 & Superior & Superior & NS & Superior & NS & NS & NS & - \\
\hline
\end{tabular}

Los cambios significativos en los límites superior e inferior se toman al menos por medio punto porcentual. NS: diferencia no significativa.

Fuente: elaboración de los autores con base en la tabla 12, $\alpha=0,50$

en cuenta el diseño muestral de las Enemdu. La tabla 14 muestra los resultados teniendo al 2007 como año base. ${ }^{8}$ Se puede observar que el incremento es estadísticamente significativo (al 95\% de confianza) al comparar las proporciones con el 2012 y el 2014. Entre el 2007 y el 2012 la proporción de

8 Se probó también los demás años posteriores al 2007 como base, pero los resultados no fueron estadísticamente significativos. 
clase media se incrementó en 2,15 puntos porcentuales, mientras que entre el 2007 y el 2014 este aumento fue de 2,59 puntos porcentuales.

Tabla 14. Cambios en la proporción de la clase media

\begin{tabular}{ccccc}
\hline Año & Coeficiente & Err. Std & $t$ & $P>|t|$ \\
\hline 2008 & 0,011 & 0,006 & 1,77 & 0,076 \\
\hline 2009 & 0,016 & 0,009 & 1,67 & 0,094 \\
\hline 2010 & 0,008 & 0,009 & 0,82 & 0,413 \\
\hline 2011 & 0,017 & 0,010 & 1,76 & 0,079 \\
\hline 2012 & 0,021 & 0,010 & 2,04 & 0,042 \\
\hline 2013 & 0,014 & 0,009 & 1,47 & 0,141 \\
\hline 2014 & 0,022 & 0,008 & 2,59 & 0,010 \\
\hline
\end{tabular}

Fuente: elaboración de los autores con base en INEc, 2007-2014 